Check for updates

Cite this: RSC Adv., 2019, 9, 34496

\title{
Designing dithienonaphthalene based acceptor materials with promising photovoltaic parameters for organic solar cells $\uparrow$
}

\begin{abstract}
Muhammad Ans, (D) *a Javed lqbal, (iD *ab ljaz Ahmad Bhatti ${ }^{a}$ and Khurshid Ayub*c
Scientists are focusing on non-fullerene based acceptors due to their efficient photovoltaic properties. Here, we have designed four novel dithienonaphthalene based acceptors with better photovoltaic properties through structural modification of a well-known experimentally synthesized reference compound $\mathrm{R}$. The newly designed molecules have a dithienonaphthalene core attached with different acceptors (end-capped). The acceptor moieties are 2-(5,6-difluoro-2-methylene-3-oxo-2,3dihydroinden-1-ylidene)malononitrile $\quad(\mathrm{H} 1), \quad 2-(5,6$-dicyano-2-methylene-3-oxo-2,3-dihydroinden-1ylidene)-malononitrile $\quad(\mathrm{H} 2), \quad$ 2-(5-methylene-6-oxo-5,6-dihydrocylopenta[c]thiophe-4-ylidene)malononitrile $(\mathrm{H} 3)$ and 2-(3-(dicyanomethylene)-2,3-dihydroinden-1-yliden)malononitrile (H4). The photovoltaic parameters of the designed molecules are discussed in comparison with those of the reference R. All newly designed molecules show a reduced HOMO-LUMO energy gap (2.17 eV to 2.28 $\mathrm{eV})$, compared to the reference $\mathrm{R}(2.31 \mathrm{eV})$. Charger transfer from donor to acceptor is confirmed by a frontier molecular orbital (FMO) diagram. All studied molecules show extensive absorption in the visible region and absorption maxima are red-shifted compared to $\mathrm{R}$. All investigated molecules have lower excitation energies which reveal high charge transfer rates, as compared to R. To evaluate the open circuit voltage, the designed acceptor molecules are blended with a well-known donor PBDB-T. The molecule $\mathrm{H} 3$ has the highest $V_{\text {oc }}$ value $(1.88 \mathrm{~V})$. TDM has been performed to show the behaviour of electronic excitation processes and electron hole location between the donor and acceptor unit. The binding energies of all molecules are lower than that of R. The lowest is calculated for $\mathrm{H} 3(0.24 \mathrm{eV})$ which reflects the highest charge transfer. The reorganization energy value for both the electrons and holes of $\mathrm{H} 2$ is lower than $\mathrm{R}$ which is indicative of the highest charge transfer rate.
\end{abstract}

Received 14th August 2019
Accepted 10th October 2019

DOI: $10.1039 / c 9 r a 06345 e$

rsc.li/rsc-advances

\section{Introduction}

The energy crisis is an ever-intensifying major challenge faced by the world today. ${ }^{1}$ According to the World Energy Council, the energy demand in 2020 will rise by $50-80 \%$ over the energy demands the world had back in $1990 .^{2}$ Fossil fuels, although depleting rapidly, have been the major energy sources since ancient times. Fossil fuels also have serious environmental impact in the form of global warming, health issues and climate change etc. These environmental issues have driven the attention of scientists towards clean and environmental friendly renewable energy sources. ${ }^{3}$ The renewable energy sources

${ }^{a}$ Department of Chemistry, University of Agriculture Faisalabad, Faisalabad, 38000, Pakistan. E-mail: ansbhatti24@gmail.com

${ }^{b}$ Punjab Bio-energy Institute, University of Agriculture, Faisalabad, 38040, Pakistan. E-mail: javedkhattak79@gmail.com

'Department of Chemistry, COMSAT University, Abbottabad Campus, Abbottabad, KPK, 22060, Pakistan. E-mail: khurshid@cuiatd.edu.pk

$\dagger$ Electronic supplementary information (ESI) available. See DOI: 10.1039/c9ra06345e include solar energy, hydro-power energy, wind energy, biomass energy. ${ }^{4}$ Among these, solar energy is a more abundant, reliable and sustainable energy source with a negligible effect on the environment. Solar cells work on the principle of the photoelectric effect (sunlight directly converts into electricity). ${ }^{5}$

Silicon is used as conducting material in the commercially available solar cells due to its high power conversion efficiency (PCE), lower toxicity, large abundance and high stability. ${ }^{6}$ Although silicon has several advantages but it also has some limitations as well such as non-tuneable energy level, brittleness, and high cost. Therefore, scientists are continuously searching for alternatives. Organic solar cells offer certain advantages such as tuneable energy levels, intensive absorption, low cost, mechanical flexibility, high reproducibility and easy processability. ${ }^{7-14}$ Organic solar cells (OSCs) contain electron donor and electron acceptors where photoexcited electron from donor shifts towards electron acceptor. ${ }^{\mathbf{1 3 , 1 5}}$ From the last two decades, fullerene based acceptors have been routinely used due to high charge mobility, high photo-induce electron and isotropic charge transfer. ${ }^{16-18}$ Although fullerene based acceptor have encouraging results, but it is tough to improve the PCE 
because of limited absorption of fullerene in visible region and less tuneability of energy levels. ${ }^{19,20}$ Recently, many nonfullerene based acceptor have been explored ${ }^{21-24}$ for their use in efficient photovoltaic materials. ${ }^{25-38}$ The acceptor molecules are rationally designed to meet the practical requirements. Among different design strategies, A-D-A (acceptor-donoracceptor) design principle has gained much more attention. The A-D-A type acceptor molecules have tune-able energy levels, broader absorption in visible region and ease in fabrication. ${ }^{39-43}$ For A-D-A strategy, the ladder type fused ring donor unit should be used in order to prevent the rotation and help in co-planarity which lowers the reorganization energy and thereby increase the charge transfer ability. ${ }^{44-46}$ Furthermore, $\mathrm{sp}^{3}$ hybridized methyl group on donor unit help in easy processability to avoid self-aggregation in blended form. Based on above strategy Zhan et al., reported A-D-A based non-fullerene acceptor, 3,9-bis-2-methylene-(3-1-dicyanomethylene)-indanone-5,5,11,11-tetrakis(4-hexylphenyl)-dithieno[2,3- $d: 2,3-d]$-sindaceno[1,2- $b: 5,6-b]$ dithiophene (ITIC), in which indacenodithienothiophene (IDTT) is used as a ladder type donor material. ${ }^{24}$ Here, we have designed four new A-D-A type acceptor molecules (H1-H4) based on IDTT donor for better performance in photovoltaic cells. The molecular structure of all molecules are illustrated in Fig. 1.

Our designed molecules contain dithienonaphthalene with methyl group as donor moiety with different end capped acceptor units. Optical properties calculated with DFT method are compared with the recently reported reference compound (DTNIC) R. ${ }^{47}$

\section{Computational details}

All calculation were performed with Gaussian 09 software. ${ }^{48}$ Gauss view 5.0 (ref. 49) was used for visualization of results. First, reference compound $\mathbf{R}$ was optimized with five different functionals of DFT including CAM-B3LYP, ${ }^{50} \mathrm{~B} 3 \mathrm{LYP},{ }^{51} \omega \mathrm{B} 97 \mathrm{XD},{ }^{52}$ PBEPBE, ${ }^{53}$ and MPW1PW91 (ref. 54) with 6-31G(d,p) basis set. For the selection of the best functional to study the electronic and optical properties of the IDTT based solar cells, absorption maxima $\left(\lambda_{\max }\right)$ of $\mathbf{R}$ are calculated with time dependent DFT with the above mentioned functionals (B3LYP, PBEPBE,

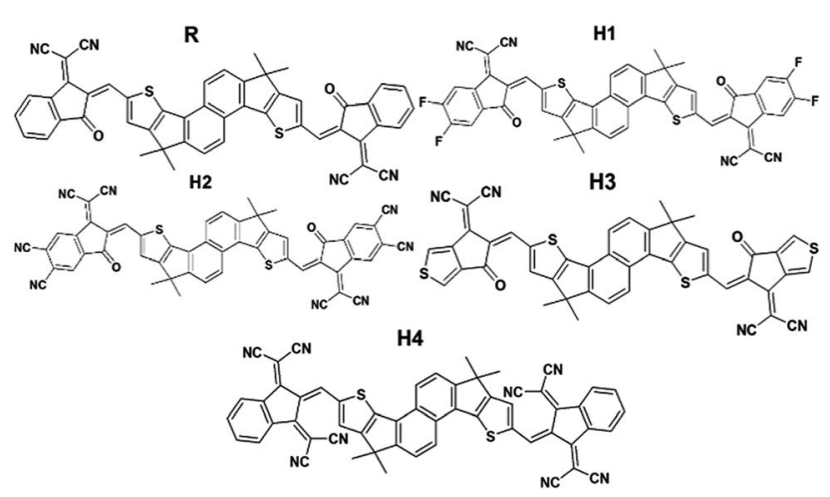

Fig. 1 Molecular structures of all molecules ( $R, H 1, H 2, H 3$, and $H 4)$.
wB97XD, CAM-B3LYP, and mPW1PW91). The $\lambda_{\max }$ values calculated with above functionals were compared with experimental $\lambda_{\max }$ value. A good agreement of $\lambda_{\max }$ value was achieved by B3LYP at 6-31G(d,p) basis set. The observation is consistent with many of our previous studies where we have shown that electronic properties are best studied with B3LYP functional. Based on this small benchmarking, all remaining calculations of the designed molecules (H1-H4) were performed with B3LYP functional. The absorption spectra were plotted in both gas and solvent phase. IEFPC model is used for calculations in the solvent phase. ${ }^{55}$ Origin 6.0 software was used for plotting UV/ visible absorption spectra. For the calculation of charge transfer, frontier molecular orbital, density of state and transition density matrix of $\mathbf{R}$ and designed molecules (H1-H4), the selected functional B3LYP was used. Density of state (DOS) spectra were plotted with Pymolyze software.

The reorganization energies were calculated for estimating charge mobilities. The reorganization energy has two parts; internal $\left(\lambda_{i}\right)$ and external $\left(\lambda_{\text {ext }}\right)$ reorganization energy. The $\lambda_{i}$ deal with quick changes in internal structure while external $\lambda_{\text {ext }}$ deals with effect of polarization and environmental relaxation. In the current study, external $\left(\lambda_{\text {ext }}\right)$ reorganization energy is neglected and we only dealt with internal reorganization energy. The mathematical equations ${ }^{56,57}$ for the calculations of reorganization energies of electron $\left(\lambda_{\mathrm{e}}\right)$ and hole $\left(\lambda_{\mathrm{h}}\right)$ are:

$$
\begin{aligned}
& \lambda_{\mathrm{e}}=\left[E_{0}{ }^{-}-E_{-}\right]+\left[E_{-}^{0}-E_{0}\right] \\
& \lambda_{\mathrm{h}}=\left[E_{0}^{+}-E_{+}\right]+\left[E_{+}^{0}-E_{0}\right]
\end{aligned}
$$

$E_{-}^{0}$ and $E_{+}^{0}$ are the energies of neutral molecules at the anion and cation state respectively. $E_{+}, E_{-}$indicates the energies of cation and anion respectively via optimized geometry of cation and anion molecules. $E_{0}{ }^{+}, E_{0}{ }^{-}$are the energies cation and anion with optimized structure of neutral molecules. Finally, $E_{0}$ is the energy of neutral molecules at ground state.

\section{Results and discussion}

The focus of this study is to design new A-D-A type acceptor molecules based on dithienonaphthalene donor unit for better performance in OSCs. The end-capped acceptors are 2-(5,6difluoro-2-methylene-3-oxo-2,3-dihydroinden-1-ylidene)malononitrile (H1), 2-(5,6-dicyano-2-methylene-3-oxo-2,3-dihydroinden-1ylidene)-malononitrile (H2), 2-(5-methylene-6-oxo-5,6-(dihydrocylopenta[ $c]$ thiophe-4-ylidene)malononitrile (H3) and 2-(3-(dicyanomethylene)-2,3-dihydroinden-1-yliden)malononitrile (H4). Initially the absorption maxima of $\mathbf{R}$ was evaluated with five different functionals (B3LYP, mPW1PW91, PBEPBE, $\omega$ B97XD and CAMB3LYP) at $6-31 G(d, p)$ basis set. The $\lambda_{\max }$ values of $\mathbf{R}$ at B3LYP, $\omega B 97 X D$, PBEPBE, CAM-B3LYP and MPW1PW91 are $607.8 \mathrm{~nm}$, $470.82 \mathrm{~nm}, 781.0 \mathrm{~nm}, 484.16 \mathrm{~nm}$, and $575.50 \mathrm{~nm}$, respectively. The reported value for $\mathbf{R}$ is $634.0 \mathrm{~nm} .{ }^{47}$ The comparison bar chart of all functionals with experimental value is given in Fig. 2 .

From Fig. 2, it is evident that the absorption maximum calculated at B3LYP/6-31G(d,p) is in best agreement with 


\section{Comparison Chart}

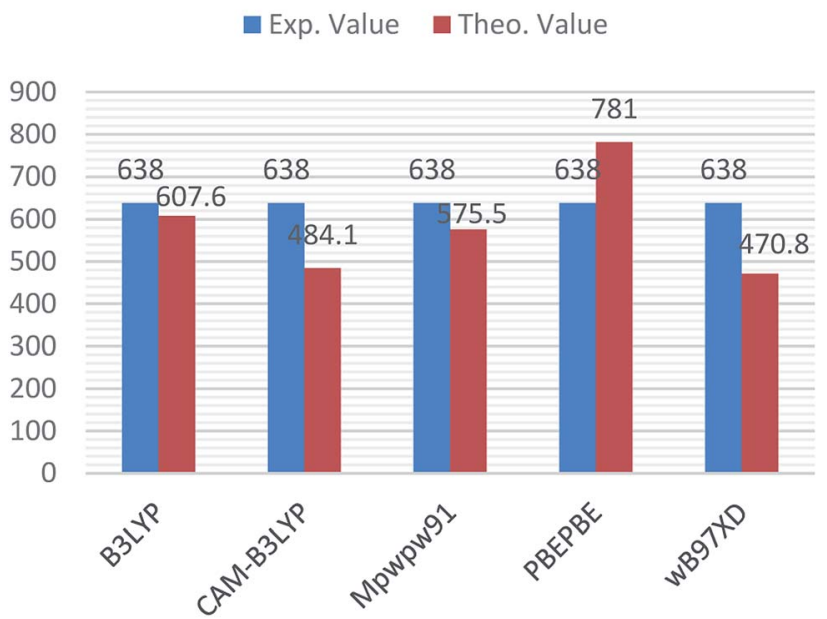

Fig. 2 Comparison diagram of $\lambda_{\max }$ value of $\mathrm{R}$ with $\omega B$ B7XD, B3LYP, CAM-B3LYP, PBEPBE and MPW1PW91 at 6-31G(d,p).

experimental value. Therefore, B3LYP/6-31G(d,p) is selected for all remaining calculations.

\section{Frontier molecular orbital diagram}

The molecular structures of designed molecules $(\mathbf{H 1}, \mathbf{H 2}, \mathbf{H} 3$ and $\mathbf{H 4}$ ) and the reference compound $\mathbf{R}$ are illustrated in Fig. 1, and their optimized geometries are presented in Fig. 3.

From the optimized geometries, it is apparent that the acceptor and the dithienonaphthalene donor unit are in one plane (see side view in Fig. 3). Two methyl groups on donor part helps to avoid self-aggregation. It is clear from Fig. 3, all studied molecules exhibits planar structure which is much preferred for high charge mobilities. ${ }^{58}$ Charger transfer is greatly influenced

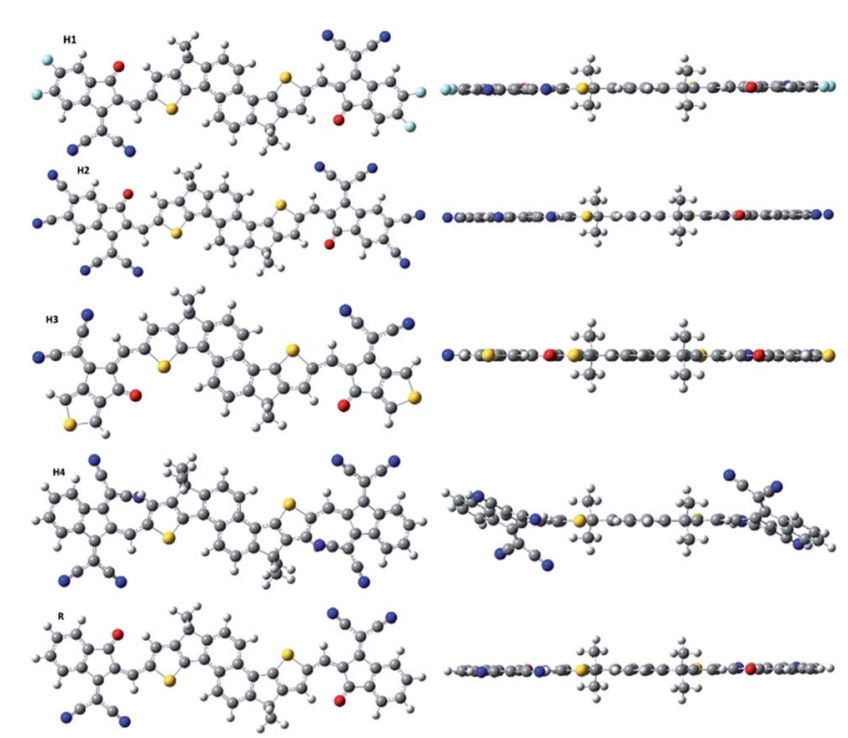

Fig. 3 Systematic optimized geometry of $\mathrm{H} 1-\mathrm{H} 4$ including model $\mathrm{R}$ at B3LYP with 6-31G $(d, p)$ level of DFT. by the distribution pattern of frontier molecular orbitals (HOMO and LUMO). The distribution of densities in HOMO and LUMO of the reference and designed molecules are illustrated in Fig. 4. In photovoltaic materials, the energy of HOMO, energy of LUMO and HOMO-LUMO energy gap (energy gap = $\left.E_{\mathrm{Lumo}}-E_{\text {Homo }}\right)$ of the organic material play key role. The energy gap dictates the power conversion efficiency, stability, chemical hardness, softness, lower dissociation energy, and binding energy which help in charger transfer analysis. ${ }^{59}$ The computed HOMO and LUMO values of $\mathbf{R}$ are $-5.68 \mathrm{eV}$ and $-3.37 \mathrm{eV}$, respectively which lead to $\mathrm{H}-\mathrm{L}$ gap of $2.31 \mathrm{eV}$. The energies of HOMO of H1, H2, H3 and $\mathbf{H 4}$ are $-5.82,-6.23$, -5.71 and $-5.86 \mathrm{eV}$, respectively. The energies of LUMO of $\mathbf{H 1}$, H2, H3 and $\mathbf{H 4}$ are $-3.54,-4.05,-3.45$ and $-3.69 \mathrm{eV}$, respectively. Form above results, it is obvious that the reference $\mathbf{R}$ has higher energies of HOMO and LUMO with respect to the designed molecules.

Among all studied molecules (R, H1-H4), H2 exhibits the lowest energies of HOMO and LUMO. The stabilized HOMO and LUMO in $\mathbf{H} \mathbf{2}$ are due to strong electron withdrawing effect of the acceptor moiety namely 5,6-dicyano-2-methylene-3-oxo-2,3dihydroinden-1-yliden-malononitrile. The HOMO and LUMO energies of $\mathbf{H 4}$ are lower than those of R, H1 and H3, which illustrates the strong electron withdrawing effect of acceptor in H4. Among all designed molecules, H3 shows high energies of HOMO and LUMO values but these values are still lower than HOMO and LUMO values of $\mathbf{R}$, which reveal that the acceptor in H3 has strong electron withdrawing effect than that of the reference $\mathbf{R}$.

The HOMO values of all designed molecules lie in the order of $\mathbf{R}>\mathbf{H 3}>\mathbf{H 1}>\mathbf{H 4}>\mathbf{H} 2$ whereas the order for the corresponding LUMO energies is R $>\mathbf{H 3}>\mathbf{H 1}>\mathbf{H 4}>\mathbf{H 2}$. Another promising key factor which effect the efficiency of organic solar cells is energy gap $\left(E_{\mathrm{g}}\right)$. The HOMO-LUMO energy values and $\mathrm{H}-\mathrm{L}$ energy gap are illustrated in Table 1 . The highest energy

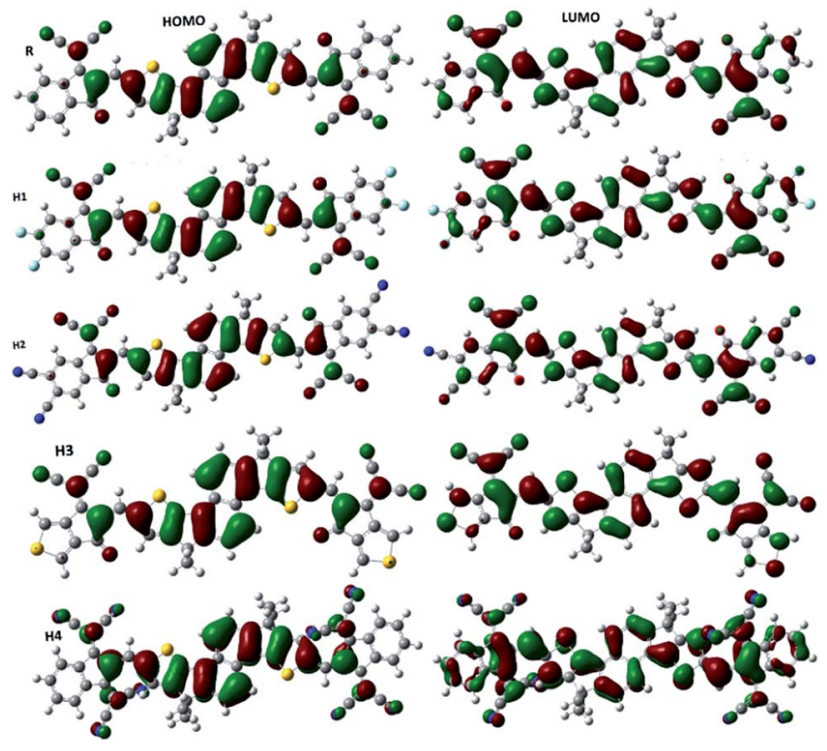

Fig. 4 HOMO-LUMO distribution pattern of $\mathrm{H} 1-\mathrm{H} 4$ and model $\mathrm{R}$. 
Table 1 HOMO, LUMO energies value with its gap at B3LYP/6$31 \mathrm{G}(\mathrm{d}, \mathrm{p})$ at $\mathrm{DFT}^{a}$

\begin{tabular}{llll}
\hline Molecules & $E_{\text {номо }}(\mathrm{eV})$ & $E_{\text {LUмо }}(\mathrm{eV})$ & $E_{\mathrm{g}}(\mathrm{eV})$ \\
\hline $\mathbf{R}$ & -5.68 & -3.37 & 2.31 \\
$\mathbf{H 1}$ & -5.82 & -3.54 & 2.28 \\
$\mathbf{H 2}$ & -6.23 & -4.05 & 2.18 \\
$\mathbf{H 3}$ & -5.71 & -3.45 & 2.25 \\
$\mathbf{H 4}$ & -5.86 & -3.69 & 2.17 \\
${ }^{a} E_{\mathrm{g}}=$ energy band gap. & &
\end{tabular}

gap is observed for $\mathbf{R}(2.31 \mathrm{eV})$. It is clear from Table 1 that the energy gaps of the designed molecules $(\mathbf{H 1}-\mathbf{H 4})$ are lower than that of the reference $\mathbf{R}$. The energy gaps of all studied molecules are in the range of 2.17 to $2.28 \mathrm{eV}$. The energy gap of $\mathbf{H 1}, \mathbf{H 2}, \mathbf{H 3}$ and $\mathbf{H 4}$ are 2.28, 2.18, 2.25 and $2.17 \mathrm{eV}$, respectively. The designed molecules $\mathbf{H 2}$ and $\mathbf{H 4}$ have comparable energy gaps. The lower energy gap of $\mathbf{H 3}$ than that of $\mathbf{H 1}$ indicates that $\mathbf{H 3}$ may have red shifts in the absorption spectrum, which mean possible better photo-absorption ability and higher short-circuit current density. The graph of HOMO-LUMO with their energy gap have been illustrated in Fig. 5 .

Furthermore, partial density of state (PDOS) were calculated to explore the optical and electronic properties of OSCs. PDOS support the facts (frontier molecular orbital diagram) which are described in Fig. 4. It is clear form Fig. 4 that the end-capped acceptor unit in the designed molecules effect the distribution pattern around HOMO and LUMO. In case of $\mathbf{R}$, the HOMO is primarily distributed on donor unit while LUMO is spread on the entire molecule. A similar type of pattern is observed for $\mathbf{H 1}$ and $\mathbf{H 2}$, where the HOMO is spread on donor core unit with small densities on the acceptor moieties whereas the LUMO is populated mostly on end capped acceptor groups with small densities present on the central donor group. For H3, the distribution of density in HOMO is comparable to those of $\mathbf{H 1}$ and $\mathbf{H} 2$ (distributed on the donor part). The distribution of
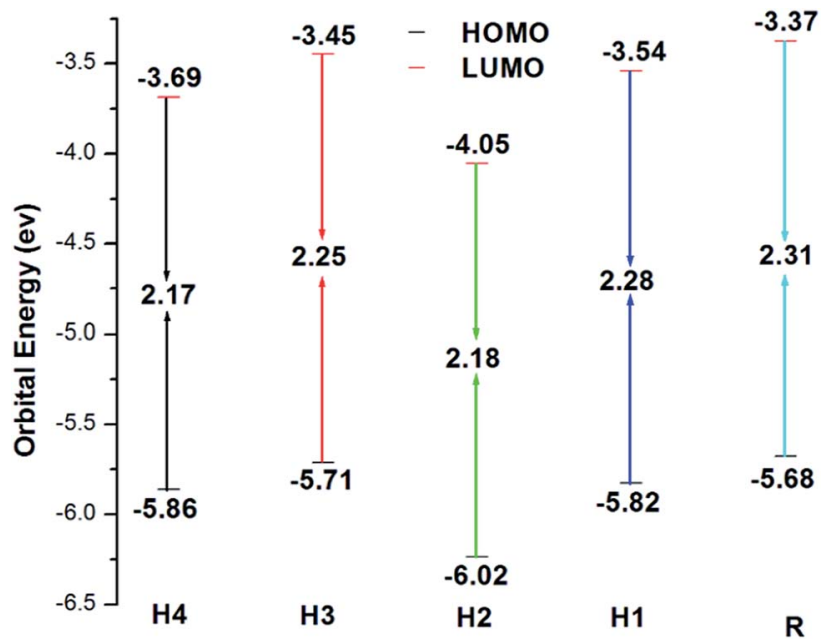

Fig. 5 Graph of HOMO-LUMO energies values of R and $(\mathrm{H} 1-\mathrm{H} 4)$ at B3LYP/6-31G(d,p) level of DFT. density in LUMO of $\mathbf{H 3}$ is different in the sense that it is spread on the entire molecule. Finally the HOMO of $\mathbf{H 4}$ is strongly distributed on donor unit without acceptor moiety, while LUMO is strongly available on acceptor unit with less spread on donor core unit. The calculated PDOS are illustrated in Fig. 6.

\section{Optical properties}

To evaluate the optical properties of $\mathbf{R}$ and (H1-H4), UV/visible absorption spectra are calculated in gas and solvent phases. The absorption maxima $\lambda_{\max }$, oscillator strength, excitation energy, and orbitals involved in the transition are shown in Table 2 for gas phase calculations.

The absorption maxima of R, H1-H4 lie in the range of 607.6 to $657.4 \mathrm{~nm}$. As shown in Table 2, it is apparent that all designed molecules (H1-H4) show absorbance in the visible region. The $\lambda_{\max }$ value of $\mathbf{R}$ calculated with B3LYP functional $(607.6 \mathrm{~nm})$ agrees nicely with the experimental value $(634.0 \mathrm{~nm})$. It is clearly seen that the strong electron withdrawing acceptor moieties particularly in $\mathbf{H 4}$ cause significant red shift in the absorption spectra. Among all molecules studied, H4 shows the highest $\lambda_{\text {max }}$ value $(657.4 \mathrm{~nm})$. The next highest absorption maximum is seen for $\mathbf{H 2}(642.7 \mathrm{~nm})$. The $\lambda_{\max }$ value of $\mathbf{H 1}$ and $\mathbf{H} \mathbf{3}$ are slightly higher than the absorption maxima of the reference compound R.

The red shifts in the absorption maxima of the designed molecules are attributed to extended conjugation between donor and end-capped acceptor groups. The absorption maxima are in decreasing order of $\mathbf{H 4}>\mathbf{H} \mathbf{2}>\mathbf{H 3}>\mathbf{H 1}>\mathbf{R}$. The absorption maxima of the designed molecules H1, H2, H3 and $\mathbf{H 4}$ are $5.4 \mathrm{~nm}, 35.1 \mathrm{~nm}, 8.2 \mathrm{~nm}$ and $49.8 \mathrm{~nm}$ red shifted when

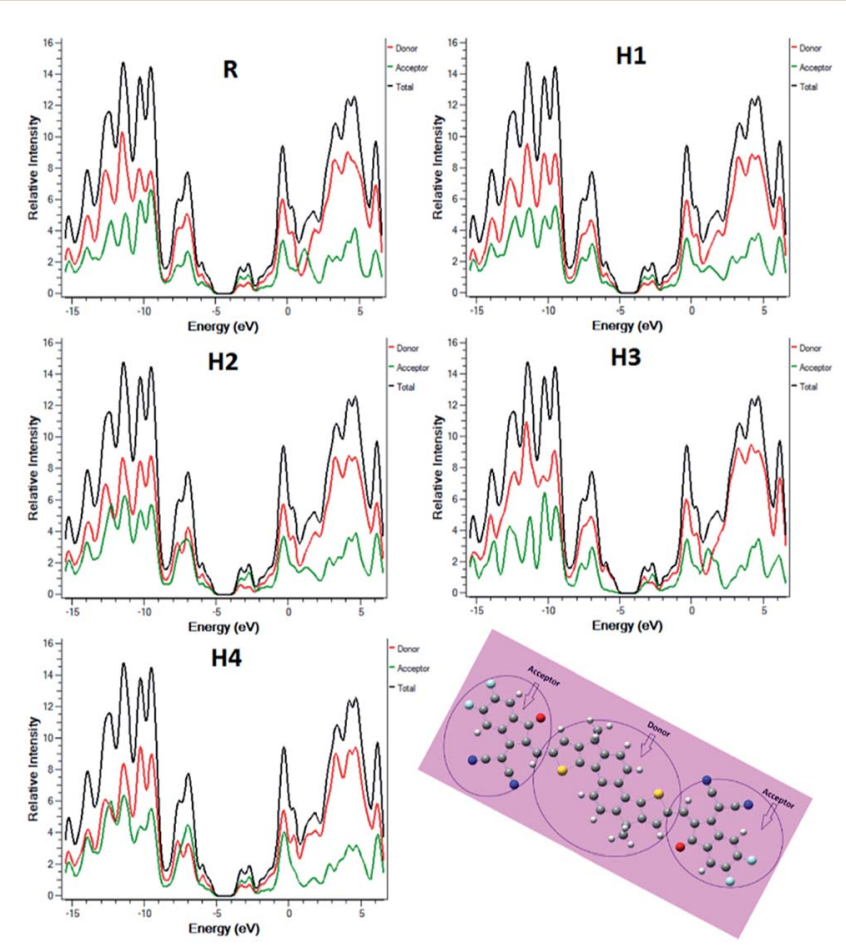

Fig. 6 Density of states around HOMOs and LUMOs of model R and $\mathrm{H} 1-\mathrm{H} 4$ at B3LYP/6-31G(d,p) level of theory. 
Table 2 The value of $\lambda_{\max }$, oscillator frequency ( $f$ ), excitation energy (eV), and assignment of R, H1, H2, H3 and H4

\begin{tabular}{llllll}
\hline & $\begin{array}{l}\text { Calcu. } \lambda_{\max } \\
\text { Compounds }\end{array}$ & $\begin{array}{l}\text { Expe. } \lambda_{\max } \\
(\mathrm{nm})\end{array}$ & $E_{\mathrm{xc}}(\mathrm{eV})$ & $f^{a}$ & Transition \\
\hline $\mathbf{R}$ & 607.6 & 634.0 & 2.04 & 1.73 & $\mathrm{H}>\mathrm{L}(+100 \%)$ \\
$\mathbf{H 1}$ & 613.0 & - & 2.02 & 1.74 & $\mathrm{H}>\mathrm{L}(+100 \%)$ \\
$\mathbf{H} 2$ & 642.7 & - & 1.93 & 1.74 & $\mathrm{H}>\mathrm{L}(+100 \%)$ \\
$\mathbf{H 3}$ & 615.8 & - & 2.01 & 1.73 & $\mathrm{H}>\mathrm{L} \mathrm{(+100 \% )}$ \\
$\mathbf{H 4}$ & 657.4 & - & 1.89 & 1.56 & $\mathrm{H}>\mathrm{L}(+99 \%)$ \\
${ }^{a}$ Oscillator strength. & & & &
\end{tabular}

compared with the $\lambda_{\max }$ value of $\mathbf{R}$. The absorption spectra are illustrated in Fig. 7.

For charge transfer, the excitation energy is an important tool. The lower the excitation energy, the greater is the charge transfer rate which ultimately increases the PCE. All studied molecules show lower excitation energy as equated to the reference $\mathbf{R}$. The excitation energy of $\mathbf{R}$ is $2.04 \mathrm{eV}$. The excitation energies of H1, H2, $\mathbf{H} \mathbf{3}$ and $\mathbf{H} \mathbf{4}$ are 2.02, 1.93, 2.01 and $1.89 \mathrm{eV}$, respectively. The incorporation of electron withdrawing group with the donor moiety in designed molecules causes lowering of excitation energies. The lowest excitation energy is calculated for $\mathbf{H 4}$ which is attributed to extended conjugation between donor and acceptor units, which leads to enhanced charge mobility. The order of excitation energies is $\mathbf{R}>\mathbf{H} \mathbf{1}>\mathbf{H} \mathbf{3}>\mathbf{H} \mathbf{2}>$ H4. The $\lambda_{\max }$ value, excitation energy, oscillator strength and transition of all molecules are also investigated in chloroform solvent with IEFPCM model and results are illustrated in Table 3.

From Table 3 it is obvious that the absorption pattern of the reference $\mathbf{R}$ and designed $\mathbf{H 1}-\mathbf{H 4}$ molecules in chloroform solvent is very similar to that in the gas phase. The $\lambda_{\max }$ values of all molecules (H1-H4) show red shift compared to the reference R. Moreover, the absorption maxima of all compounds in

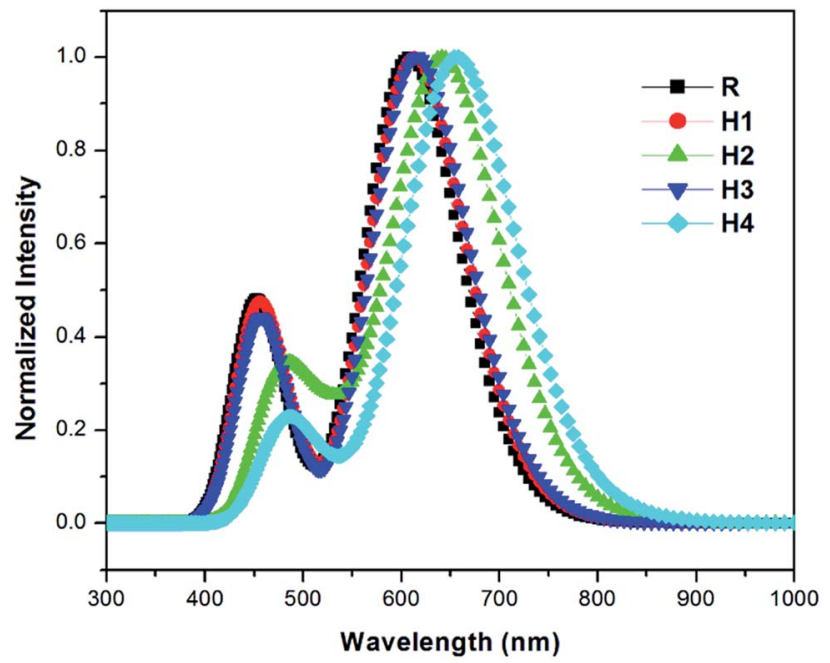

Fig. 7 Absorption spectra of $\mathrm{H} 1, \mathrm{H} 2, \mathrm{H} 3, \mathrm{H} 4$ and $\mathrm{R}$ in gas phase at TD$B 3 L Y P / 6-31 G(d, p)$.
Table 3 The wavelength $\lambda_{\max }$, excitation energy (eV), assignment and oscillator frequency $(f)$ of $\mathrm{R}, \mathrm{H} 1, \mathrm{H} 2, \mathrm{H} 3$ and $\mathrm{H} 4$ in chloroform solvent

\begin{tabular}{|c|c|c|c|c|c|}
\hline Compounds & $\begin{array}{l}\text { Calcu. } \lambda_{\max } \\
(\mathrm{nm})\end{array}$ & $\begin{array}{l}\text { Expe. } \lambda_{\max } \\
(\mathrm{nm})\end{array}$ & $E_{\mathrm{xc}}(\mathrm{eV})$ & $f^{a}$ & Transition \\
\hline $\mathbf{R}$ & 652.2 & 634 & 1.90 & 2.05 & $\mathrm{H}>\mathrm{L}(+99 \%)$ \\
\hline H1 & 659.7 & - & 1.88 & 2.05 & $\mathrm{H}>\mathrm{L}(+99 \%)$ \\
\hline H2 & 721 & - & 1.72 & 2.12 & $\mathrm{H}>\mathrm{L}(+99 \%)$ \\
\hline H3 & 659.9 & - & 1.88 & 2.09 & $\mathrm{H}>\mathrm{L}(+99 \%)$ \\
\hline $\mathbf{H 4}$ & 704.9 & - & 1.76 & 1.86 & $\mathrm{H}>\mathrm{L}(+98 \%)$ \\
\hline
\end{tabular}

chloroform solvent are red shifted compared to those in the gas phase. For example, the absorption maximum of $\mathbf{R}$ in chloroform solvent is $652.2 \mathrm{~nm}$ compared to $607.6 \mathrm{~nm}$ in the gas phase. Similarly, the $\lambda_{\max }$ value of $\mathbf{H 1}, \mathbf{H} 2, \mathbf{H} \mathbf{3}$ and $\mathbf{H 4}$ are $659.7 \mathrm{~nm}, 721 \mathrm{~nm}, 659.0 \mathrm{~nm}$ and $704.9 \mathrm{~nm}$, respectively. The $\lambda_{\max }$ values of $\mathbf{H 1}, \mathbf{H 2}, \mathbf{H} \mathbf{3}$ and $\mathbf{H 4}$ are red shifted (from those in the gas phase) by $46.7 \mathrm{~nm}, 78.3 \mathrm{~nm}, 44.1 \mathrm{~nm}$, and $47.7 \mathrm{~nm}$, respectively (Fig. 8).

In brief, the absorption maxima of our designed molecules are more red shifted (in both gas as well as in chloroform solvent) than the reference compound $\mathbf{R}$ which is highly beneficial for their application in photovoltaic cells.

\section{Charge mobilities}

The performance of OSCs is directly related to charge mobilities which can be evaluated through reorganization energies of electron $\left(\lambda_{\mathrm{e}}\right)$ and hole $\left(\lambda_{\mathrm{h}}\right)$. Reorganization energy is calculated with selected functional and the results are summarized in Table 4.

Charge mobilities and reorganization energies are in inverse relationship. There is inverse relationship between reorganization energies and charge mobilities. The lower the

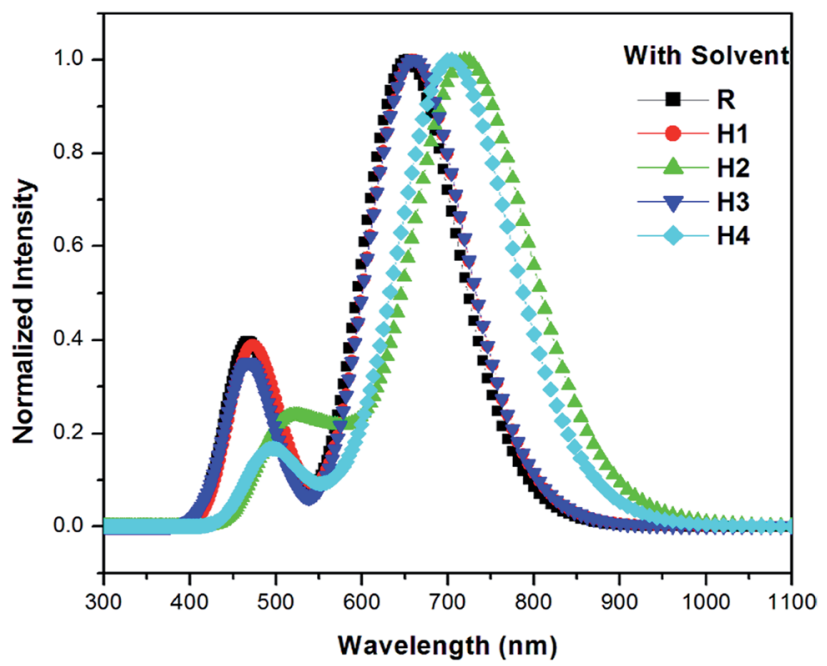

Fig. 8 UV/visible spectra of all molecules in solvent (chloroform) at TD-B3LYP/6-31G(d,p) level of DFT. 
Table 4 Reorganization energy of model $R$ and selected molecules $(\mathrm{H} 1-\mathrm{H} 4)$

\begin{tabular}{llr}
\hline Molecule & $\lambda_{\mathrm{e}}{ }^{a}(\mathrm{eV})$ & $\lambda_{\mathrm{h}}{ }^{b}(\mathrm{eV})$ \\
\hline R & $5.939 \times 10^{-3}$ & $4.648 \times 10^{-3}$ \\
H1 & $6.065 \times 10^{-3}$ & $4.985 \times 10^{-3}$ \\
H2 & $4.019 \times 10^{-3}$ & $4.718 \times 10^{-3}$ \\
H3 & $5.614 \times 10^{-3}$ & $4.546 \times 10^{-3}$ \\
H4 & $1.326 \times 10^{-2}$ & $5.89 \times 10^{-3}$ \\
${ }^{a}$ Electron reorganization. ${ }^{b}$ Hole reorganization. &
\end{tabular}

reorganization energy, the higher is the charge mobility. ${ }^{60}$ Our designed molecules shows lower reorganization energies value as compared to recently reported molecule namely SBF1 (ref. 61) with highest absorption value $400 \mathrm{~nm}$. The reorganization energy for SBF1 is $0.144 \mathrm{eV}$. It is clear from above Table 4 our designed molecules have high charge mobilities due to lower value of reorganization energies.

Reorganization energies are divided into two parts; internal reorganization and external reorganization energy. External reorganization energy deals with environmental relaxation while internal reorganization energy deals with changes in the geometries of cation and anion. Cationic geometry describes with the mobility of cation while anionic geometry deals with the mobility of electron from donor material. In the current study, we are only dealing with internal reorganization energy.

Intra molecular reorganization energy is estimated with the aid of Marcus equation. ${ }^{62}$ It is the energy cost due to geometry form charge to neutral and neutral to charge molecules. Charge mobilities of organic solar cells is control by reorganization energies. The Marcus equation is given below

$$
\begin{aligned}
k_{\mathrm{ET}} & =\left(\frac{4 \pi^{2}}{h}\right) \times t^{2}\left(\frac{1}{4 \pi \lambda_{\mathrm{s}} k T}\right)^{1 / 2} \\
& \times \sum_{\nu^{\prime}} \exp (-S) \frac{S \nu^{\prime}}{\nu^{\prime}} \exp \left(\frac{\left(\Delta G_{\mathrm{o}}+\lambda_{\mathrm{s}}+\nu^{\prime} \hbar\langle W \nu\rangle\right)^{2}}{4 \pi \lambda_{\mathrm{s}} k T}\right)
\end{aligned}
$$

where, $k_{\mathrm{ET}}$ is the charge mobility of electron, electronic coupling between the states is represented by $T, \Delta G_{\mathrm{o}}$ is the Gibbs free energy change and $\lambda_{\mathrm{s}}$ is the reorganization energy

$$
S=\frac{\lambda_{\mathrm{i}}}{\hbar\langle W v\rangle}
$$

The $\lambda_{i}$ in this equation can be explain in term of reorganization energy. This equation shows that, smaller is the geometry relaxation causes high electron transfer rate $\left(k_{\mathrm{ET}}\right)$. If both geometries such as geometry of final state (neutral state) and geometry of initial state (ionized state) are same then electron can easily be passes without any occurring of vibrations. If both geometries are different then the molecule has to wait until vibrations bring the acceptor into a shape that is more similar to the donor. Charge mobility is an activated process because it requires vibration that will prepare the system so that the geometries are equivalent and the electron move.
The $\lambda_{\mathrm{e}}$ value of $\mathbf{R}$ is $0.005939 \mathrm{eV}$. The designed molecules $\mathbf{H 1}$ and $\mathbf{H 4}$ have higher value of electron reorganization energies which is indicative of lower charge transfer rate in these molecules. The lower value of $\lambda_{\mathrm{e}}$ for $\mathbf{H 2}$ and $\mathbf{H 3}$ means high charge transfer rate from donor to acceptor unit. Among all, the lowest value of $\mathbf{H} 2$ is due to extending conjugation in acceptor unit. The $\lambda_{\mathrm{e}}$ values are in order of $\mathbf{H 4}>\mathbf{H 1}>\mathbf{R}>\mathbf{H} \mathbf{3}>\mathbf{H} 2$.

Secondly, the hole reorganization $\left(\lambda_{h}\right)$ value of $\mathbf{R}$ is $0.004648 \mathrm{eV}$. A similar pattern is observed in hole reorganization energy; $\mathbf{H 1}$ and $\mathbf{H 4}$ have higher hole reorganization energies which mean low charge transfer rates. Among all the designed molecules, $\mathbf{H 2}$ and $\mathbf{H 3}$ exhibit lower reorganization energy with respect to $\mathbf{R}$, which reflects high charge transfer mobilities. The designed molecules H1 and H4 have high reorganization energies which indicate low charge transfer rate as compared to $\mathbf{H} \mathbf{2}$ and $\mathbf{H 3}$. Among designed molecules, the highest hole mobility is calculated for $\mathbf{H} \mathbf{2}$ which is attributed to lower reorganization energy value. The $\lambda_{\mathrm{h}}$ value of all investigated acceptors are in the order of $\mathbf{H 4}>\mathbf{H 1}>\mathbf{H 2}>\mathbf{R}>\mathbf{H 3}$. By comparing hole and electron reorganization energies, the lower value of hole reorganization energy with respect to electron shows that these molecules are good for hole carrier. Above discussion indicates that the investigated molecules are efficient photovoltaic material in OSCs.

\section{Open circuit voltage $\left(V_{o c}\right)$}

To evaluate the efficiency of solar cells, the most important factor is open circuit voltage $\left(V_{\mathrm{oc}}\right) \cdot{ }^{20} V_{\mathrm{oc}}$ describes the maximum current which can be taken out from any devices. $V_{\text {oc }}$ depends upon many factors such as charge carrier combination, external fluorescence, light source, temperature of device, and energy level of solar cells. However, $V_{\text {oc }}$ primarily depends on light generated and saturation current. The $V_{\mathrm{oc}}$ is approximately equal to the difference of LUMO (acceptor) and HOMO (donor) moiety. The value of $V_{\text {oc }}$ is calculated with the aid of Scharber equation, ${ }^{63}$ as eqn (III).

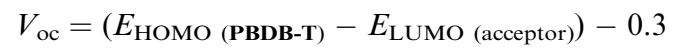

In above equation 0.3 is the empirical factor for efficient charge separation. ${ }^{64}$ To obtain the high value of $V_{\mathrm{oc}}$ the HOMO of donor unit should be low in energy while LUMO of acceptor unit should have high energy. In the current study, we designed non-fullerene based acceptor so we blended our molecules with
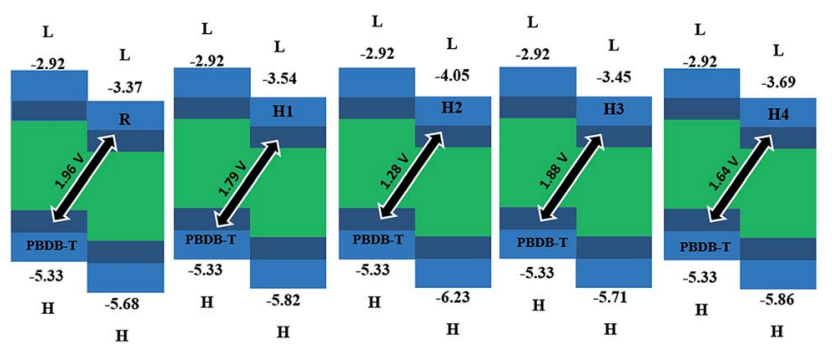

Fig. 9 The open circuit voltages $\left(V_{\text {oc }}\right)$ of reference $\mathrm{R}$ and $\mathrm{H} 1-\mathrm{H} 4$ with respect to donor material PBDB-T donor. 
well-known PBDB-T donor. The calculate $V_{\text {oc }}$ with PBDB-T are shown in Fig. 9.

The $V_{\text {oc }}$ value of $\mathbf{R}$ with respect to PBDB-T is $1.96 \mathrm{~V}$. The $V_{\mathrm{oc}}$ values of H1, H2, H3 and $\mathbf{H 4}$ are $1.79 \mathrm{~V}, 1.28 \mathrm{~V}, 1.88 \mathrm{~V}$ and $1.64 \mathrm{~V}$ respectively. The $V_{\mathrm{oc}}$ value of $\mathbf{H 1}$ is $0.51 \mathrm{~V}$ higher than $\mathbf{H 2}$. The computed $V_{\text {oc }}$ value for $\mathbf{H 3}$ is $0.24 \mathrm{~V}$ higher than that of $\mathbf{H 4}$. The designed molecules $\mathbf{H 4}$ has $V_{\text {oc }} 0.36 \mathrm{~V}$ higher value with respect to $\mathbf{H 2}$. The highest $V_{\mathrm{oc}}$ value is shown by $\mathbf{H 3}$. The $V_{\mathrm{oc}}$ values for H1, H2, H3 and H4 lies in decreasing order of H3 $>$ H1 $>$ H4 > H2.

\section{Exciton binding energy $\left(E_{\mathrm{b}}\right)$ and transition density matrix}

The nature of transition from $\mathrm{S}_{0}-\mathrm{S}_{1}$ is evaluated with the help of transition density matrix (TDM) ${ }^{65-69}$ with the selected functional. In our study, the contribution of hydrogen atom is very small therefore, it is neglected. The TDM illustrates the localization of electron and hole in the excited state, electronic excitation and also helps to understand the effect of acceptor and donor in the excited state. To evaluate the TDM, we divided our designed molecules into two parts one is donor denoted as $\mathrm{D}$ and other is end-capped acceptor moiety which is denoted as A. TDM results are shown in Fig. 10.

The designed and reference molecules show similar behaviour; the electron consistency is diagonal to donor, and on acceptor group. The electron coherence is available on donor as well as on acceptor unit. From TDM diagram, is it confirmed that the electron is transferred from donor part to acceptor unit.
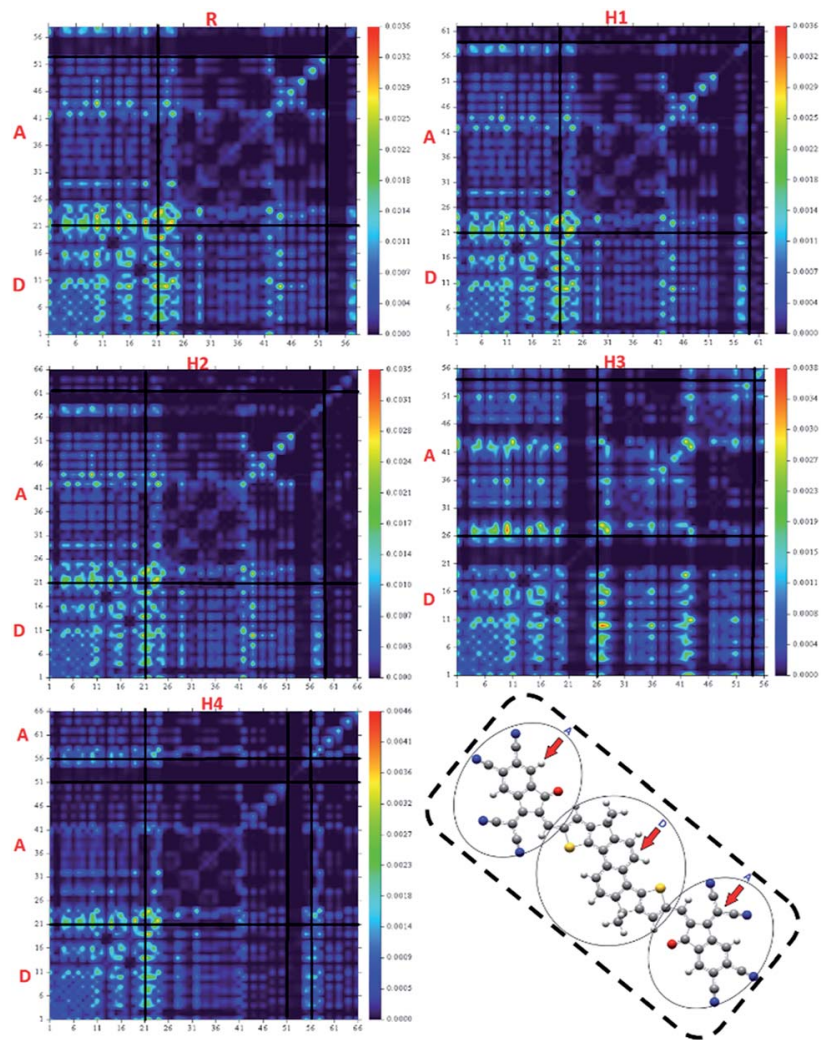

Fig. 10 Transition density matrix of reference $R$ and designed molecules $(\mathrm{H} 1-\mathrm{H} 4)$ at $\mathrm{S} 1$ state.
Additionally, the coherence interaction between donor to acceptor unit in excited state is in the decreasing order of $\mathbf{R}=$ H4 > H1 > H2 > H3. All designed molecules have lower coefficient of interaction which means that these molecules have high charge transfer rate with respect to R. Among all, the highest charge density is observed for $\mathbf{H 3}$.

The charge dissociation ability of all molecule is higher than $\mathbf{R}$ which increases current charge density $J_{\mathrm{sc}}$.

To enhance the efficiency of OSCs, the binding energy is a promising tool. Binding energy describes the coulombic interaction between positive and negative charges. Higher value of $E_{\mathrm{b}}$ mean high coulombic interaction between positive and negative charges which mean lower will be the exciton dissociation in excited state. The $E_{\mathrm{b}}$ value for $\mathbf{R}$ and designed molecules can be calculated by using following eqn (IV).

$$
E_{\mathrm{b}}=E_{\mathrm{H}-\mathrm{L}}-E_{\mathrm{opt}}
$$

In above equation $E_{\mathrm{b}}$ is the binding energy, $E_{\mathrm{H}-\mathrm{L}}$ is the energy gap and $E_{\text {opt }}$ is the single point energy and results are shown in Table 5.

Binding energy analysis support the facts described by transition density matrix in Fig. 10. The binding energy of $\mathbf{R}$, H1, H2, H3, and H4 are 0.27, 0.26, 0.26, 0.24 and $0.27 \mathrm{eV}$, respectively. $\mathbf{R}$ has highest binding energy which mean lower charge transfer. Among all studied molecules, $\mathbf{H} 3$ has the lowest binding energy which mean the highest charge transfer ability. Binding energy of reference $\mathbf{R}$ and $\mathbf{H 4}$ have same value, hence both $\mathbf{R}$ and $\mathbf{H} 4$ exhibit same charge mobilities. The binding energy of $\mathbf{H 1}$ is comparable to $\mathbf{H} \mathbf{2}$ however, these values are lower than that of $\mathbf{R}$ which reveals that both $\mathbf{H 1}$ and $\mathbf{H} 2$ show same charge transfer rate (higher than reference $\mathbf{R}$ ). Furthermore, the order of binding energies of all molecules is $\mathbf{R}=\mathbf{H 4}>$ $\mathrm{H} 1=\mathrm{H} 2>\mathrm{H} 3$.

\section{Charge transfer analysis}

To evaluate the nature of transition, we made a complex between a designed molecules (H2) and PBDB-T donor. H2 molecule is chosen due to low reorganization energy and high charge mobility. The complex H2/PBDB-T is optimized with the selected functional. The literature reveals that the dipole moment from donor to acceptor is responsible for charge transfer. ${ }^{70-73}$ The side view of optimized geometry of complex in shown in Fig. 11.

Table 5 The first singlet excitation energies $\left(E_{\mathrm{opt}}\right)$, energy gap $E_{\mathrm{H}-\mathrm{L}}$, and exciton binding energies $\left(E_{\mathrm{b}}\right)^{a}$

\begin{tabular}{llll}
\hline Molecules & $E_{\mathrm{H}-\mathrm{L}}(\mathrm{eV})$ & $E_{\text {opt }}(\mathrm{eV})$ & $E_{\mathrm{b}}(\mathrm{eV})$ \\
\hline R & 2.31 & 2.04 & 0.27 \\
H1 & 2.28 & 2.02 & 0.26 \\
H2 & 2.18 & 1.92 & 0.26 \\
H3 & 2.25 & 2.01 & 0.24 \\
H4 & 2.17 & 1.90 & 0.27
\end{tabular}

${ }^{a} E_{\mathrm{b}}=$ binding energy. 


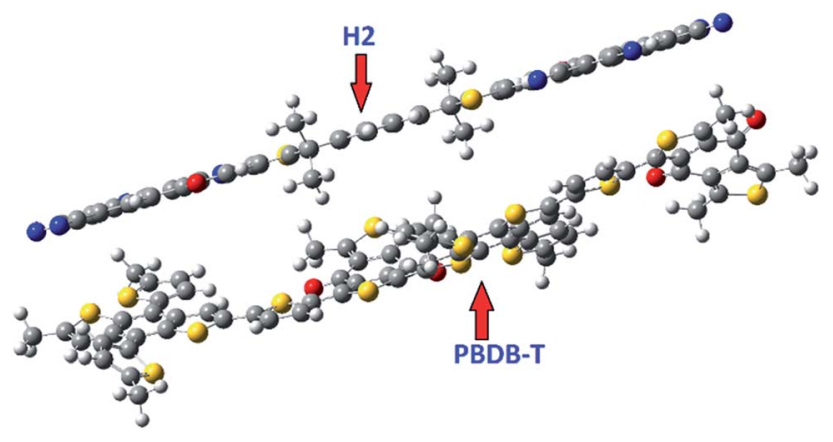

Fig. 11 Optimized geometry acceptor H2 and PBDB-T donor unit.
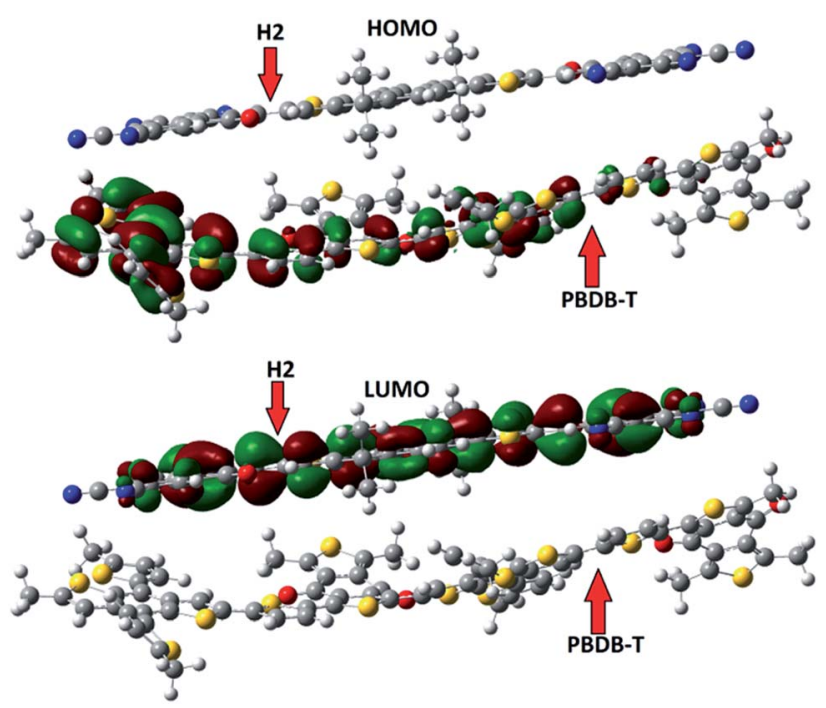

Fig. 12 The distribution pattern around (HOMO) and LUMO of $\mathrm{H} 2$ and PBDB-T.

The dipole moment $\mu$ of $\mathbf{H 2}$ /PBDB-T arises due to permanent dipole from donor to acceptor unit. To further check the nature of charge transfer (electronic properties) and distribution pattern from donor to acceptor unit, the FMOs analyses has been performed with selected functional and results are illustrated in Fig. 12. It is clear from Fig. 11 that the charge density is shifted from donor to acceptor unit which is in great agreement to show the nature of charges. In the complex, the HOMO is mainly distributed on donor unit (PBDB-T) while LUMO is spread on acceptor moiety.

\section{Conclusions}

In this study, we have designed four novel dithienonaphthalene based acceptor molecules (H1-H4) with different acceptor moieties to promote the opto-electronic properties of OSCs. By increasing extending conjugation in acceptor unit with central donor skeleton enhances, opto-electronic properties of OSCs are enhanced. A suitable functional for all further calculations is selected through a benchmark study where UV-vis spectrum of the reference compound $\mathbf{R}$ is calculated with five different functionals and compared with the experimental data. After careful assessment, B3LYP/6-31G(d,p) is chosen for further calculations. All molecules shows red shift in visible region with respect to reference molecule $\mathbf{R}$. Among all, $\mathbf{H} 2$ has maximum red shift in the absorption spectrum $(721 \mathrm{~nm})$ in chloroform solvent. All designed molecules have reduced HOMO-LUMO energy gap however, the molecule $\mathbf{H 4}$ has the lowest HOMOLUMO energy gap which is attributed to extended conjugation in acceptor moiety. Moreover, all designed molecules have higher charge transfer rate with respect to $\mathbf{R}$, (lower value of excitation energy) where $\mathbf{H} 2$ has the highest charge transfer rate. For $V_{\mathrm{oc}}$, the designed molecules are scaled up with donor PBDB-T. The highest $V_{\text {oc }}$ value is calculated for H3. To calculate the charge transfer, binding energies are calculated which illustrate that all newly designed molecules have lower value of binding energies (high excitation and high charge transfer rate). The highest charge mobility is calculated for $\mathbf{H} 2$ which is due to lowest value of binding energy. To show the nature of charge transfer from donor to acceptor, the designed molecule $\mathbf{H 2}$ is blended with PBDB-T donor group. In summary, all designed molecules have outstanding opto-electronic properties with respect to reference molecule $\mathbf{R}$.

\section{Conflicts of interest}

There are no conflicts to declare.

\section{Acknowledgements}

The Computations/simulations/SIMILAR were performed on resources provided by the Swedish National Infrastructure for Computing (SNIC) at Umeå University, 901 87, Umeå, Sweden. The authors acknowledge the financial and technical support from Punjab Bio-energy Institute (PBI), University of Agriculture Faisalabad (UAF).

\section{Notes and references}

1 D. M. Chapin, C. Fuller and G. Pearson, J. Appl. Phys., 1954, 25, 676-677.

2 A. M. Omer, Renewable Sustainable Energy Rev., 2008, 12, 2265-2300.

3 M. D. Archer and M. A. Green, Clean electricity from photovoltaics, World Scientific, 2001.

4 R. Prakash and I. K. Bhat, Renewable Sustainable Energy Rev., 2009, 13, 2716-2721.

5 G. P. Smestad, Sol. Energy Mater. Sol. Cells, 2004, 82, 227-240. 6 A. McEvoy and T. Markvart, Solar cells: materials, manufacture and operation, Academic Press, 2012.

7 A. Facchetti, Chem. Mater., 2010, 23, 733-758.

8 H. Yao, L. Ye, H. Zhang, S. Li, S. Zhang and J. Hou, Chem. Rev., 2016, 116, 7397-7457.

9 Y. Li, Acc. Chem. Res., 2012, 45, 723-733.

10 J. Chen and Y. Cao, Acc. Chem. Res., 2009, 42, 1709-1718.

11 M. Ans, J. Iqbal, B. Eliasson and K. Ayub, Comput. Mater. Sci., 2019, 159, 150-159. 
12 Y. Lin, Y. Li and X. Zhan, Chem. Soc. Rev., 2012, 41, 42454272.

13 Y.-J. Cheng, S.-H. Yang and C.-S. Hsu, Chem. Rev., 2009, 109, 5868-5923.

14 G. Yu, J. Gao, J. C. Hummelen, F. Wudl and A. J. Heeger, Science, 1995, 270, 1789-1791.

15 Y. Chen, X. Wan and G. Long, Acc. Chem. Res., 2013, 46, 2645-2655.

16 A. J. Heeger, Adv. Mater., 2014, 26, 10-28.

17 Y. Ma, Z. Kang and Q. Zheng, J. Mater. Chem. A, 2017, 5, 1860-1872.

18 M. Ans, J. Iqbal, K. Ayub, E. Ali and B. Eliasson, Mater. Sci. Semicond. Process., 2019, 94, 97-106.

19 X. Zhan, A. Facchetti, S. Barlow, T. J. Marks, M. A. Ratner, M. R. Wasielewski and S. R. Marder, Adv. Mater., 2011, 23, 268-284.

20 M. Ans, K. Ayub, I. A. Bhatti and J. Iqbal, $R S C A d v .$, 2019, 9, 3605-3617.

21 C. B. Nielsen, S. Holliday, H.-Y. Chen, S. J. Cryer and I. McCulloch, Acc. Chem. Res., 2015, 48, 2803-2812.

22 C. Zhan and J. Yao, Chem. Mater., 2016, 28, 1948-1964.

23 M. Ans, J. Iqbal, Z. Ahmad, S. Muhammad, R. Hussain, B. Eliasson and K. Ayub, ChemistrySelect, 2018, 3, 1279712804.

24 Y. Lin, J. Wang, Z. G. Zhang, H. Bai, Y. Li, D. Zhu and X. Zhan, Adv. Mater., 2015, 27, 1170-1174.

25 Y. Zhong, M. T. Trinh, R. Chen, G. E. Purdum, P. P. Khlyabich, M. Sezen, S. Oh, H. Zhu, B. Fowler and B. Zhang, Nat. Commun., 2015, 6, 8242.

26 Y. J. Hwang, H. Li, B. A. Courtright, S. Subramaniyan and S. A. Jenekhe, Adv. Mater., 2016, 28, 124-131.

27 D. Meng, D. Sun, C. Zhong, T. Liu, B. Fan, L. Huo, Y. Li, W. Jiang, H. Choi and T. Kim, J. Am. Chem. Soc., 2015, 138, 375-380.

28 S. Holliday, R. S. Ashraf, A. Wadsworth, D. Baran, S. A. Yousaf, C. B. Nielsen, C.-H. Tan, S. D. Dimitrov, Z. Shang and N. Gasparini, Nat. Commun., 2016, 7, 11585.

29 F. Liu, Z. Zhou, C. Zhang, T. Vergote, H. Fan, F. Liu and X. Zhu, J. Am. Chem. Soc., 2016, 138, 15523-15526.

30 S. Holliday, R. S. Ashraf, C. B. Nielsen, M. Kirkus, J. A. Röhr, C.-H. Tan, E. Collado-Fregoso, A.-C. Knall, J. R. Durrant and J. Nelson, J. Am. Chem. Soc., 2015, 137, 898-904.

31 X. F. Wu, W. F. Fu, Z. Xu, M. Shi, F. Liu, H. Z. Chen, J. H. Wan and T. P. Russell, Adv. Funct. Mater., 2015, 25, 5954-5966.

32 M. Ans, J. Iqbal, B. Eliasson, M. J. Saif, H. M. A. Javed and K. Ayub, J. Mol. Model., 2019, 25, 129.

33 Y. Kim, C. Song, E.-J. Ko, D. Kim, S.-J. Moon and E. Lim, RSC $A d v .$, 2015, 5, 4811-4821.

34 P. Sonar, G.-M. Ng, T. T. Lin, A. Dodabalapur and Z.-K. Chen, J. Mater. Chem., 2010, 20, 3626-3636.

35 D. Meng, H. Fu, C. Xiao, X. Meng, T. Winands, W. Ma, W. Wei, B. Fan, L. Huo and N. L. Doltsinis, J. Am. Chem. Soc., 2016, 138, 10184-10190.

36 Y. Liu, C. Mu, K. Jiang, J. Zhao, Y. Li, L. Zhang, Z. Li, J. Y. L. Lai, H. Hu and T. Ma, Adv. Mater., 2015, 27, 10151020 .
37 P. E. Hartnett, A. Timalsina, H. R. Matte, N. Zhou, X. Guo, W. Zhao, A. Facchetti, R. P. Chang, M. C. Hersam and M. R. Wasielewski, J. Am. Chem. Soc., 2014, 136, 1634516356.

38 B. Xiao, A. Tang, J. Zhang, A. Mahmood, Z. Wei and E. Zhou, Adv. Energy Mater., 2017, 7, 1602269.

39 M. Cheng, C. Chen, X. Yang, J. Huang, F. Zhang, B. Xu and L. Sun, Chem. Mater., 2015, 27, 1808-1814.

40 D. Baran, R. S. Ashraf, D. A. Hanifi, M. Abdelsamie, N. Gasparini, J. A. Röhr, S. Holliday, A. Wadsworth, S. Lockett and M. Neophytou, Nat. Mater., 2017, 16, 363.

41 F. Zhao, S. Dai, Y. Wu, Q. Zhang, J. Wang, L. Jiang, Q. Ling, Z. Wei, W. Ma and W. You, Adv. Mater., 2017, 29, 1700144.

42 B. Kan, H. Feng, X. Wan, F. Liu, X. Ke, Y. Wang, Y. Wang, H. Zhang, C. Li and J. Hou, J. Am. Chem. Soc., 2017, 139, 4929-4934.

43 N. Qiu, H. Zhang, X. Wan, C. Li, X. Ke, H. Feng, B. Kan, H. Zhang, Q. Zhang and Y. Lu, Adv. Mater., 2017, 29, 1604964.

44 Y. Ma, Q. Zheng, Z. Yin, D. Cai, S.-C. Chen and C. Tang, Macromolecules, 2013, 46, 4813-4821.

45 Y. Ma, S.-C. Chen, Z. Wang, W. Ma, J. Wang, Z. Yin, C. Tang, D. Cai and Q. Zheng, Nano Energy, 2017, 33, 313-324.

46 J. Lee, A. J. Kalin, T. Yuan, M. Al-Hashimi and L. Fang, Chem. Sci., 2017, 8, 2503-2521.

47 Y. Ma, M. Zhang, Y. Yan, J. Xin, T. Wang, W. Ma, C. Tang and Q. Zheng, Chem. Mater., 2017, 29, 7942-7952.

48 M. Frisch, G. Trucks, H. Schlegel, G. Scuseria, M. Robb, J. Cheeseman, G. Scalmani, V. Barone, B. Mennucci and G. Petersson, J. Exp. Clin. Med., 2009, 4(3), 165-169.

49 R. D. Dennington, T. A. Keith and J. M. Millam, Gaussian Inc, 2008.

50 T. Yanai, D. P. Tew and N. C. Handy, Chem. Phys. Lett., 2004, 393, 51-57.

51 B. Civalleri, C. M. Zicovich-Wilson, L. Valenzano and P. Ugliengo, CrystEngComm, 2008, 10, 405-410.

52 J.-D. Chai and M. Head-Gordon, Phys. Chem. Chem. Phys., 2008, 10, 6615-6620.

53 R. M. El-Shishtawy, A. M. Asiri, S. G. Aziz and S. A. Elroby, J. Mol. Model., 2014, 20, 2241.

54 C. Adamo and V. Barone, J. Chem. Phys., 1998, 108, 664-675. 55 M. Cossi, V. Barone, B. Mennucci and J. Tomasi, Chem. Phys. Lett., 1998, 286, 253-260.

56 M. Irfan, J. Iqbal, S. Sadaf, B. Eliasson, U. A. Rana, S. Ud-din Khan and K. Ayub, Int. J. Quantum Chem., 2017, 117, e25363.

57 M. E. Köse, W. J. Mitchell, N. Kopidakis, C. H. Chang, S. E. Shaheen, K. Kim and G. Rumbles, J. Am. Chem. Soc., 2007, 129, 14257-14270.

58 G. Zhang, G. Yang, H. Yan, J. H. Kim, H. Ade, W. Wu, X. Xu, Y. Duan and Q. Peng, Adv. Mater., 2017, 29, 1606054.

59 S. S. Amiri, S. Makarem, H. Ahmar and S. Ashenagar, J. Mol. Struct., 2016, 1119, 18-24.

60 B. Xu, D. Bi, Y. Hua, P. Liu, M. Cheng, M. Grätzel, L. Kloo, A. Hagfeldt and L. Sun, Energy Environ. Sci., 2016, 9, 873-877.

61 B. Zhang, Y. Xu, J. Yang, Y. Liao, L. Tong and S. Zhou, Comput. Theor. Chem., 2019, 112575.

62 R. A. Marcus, Annu. Rev. Phys. Chem., 1964, 15, 155-196. 
63 M. C. Scharber, D. Mühlbacher, M. Koppe, P. Denk, C. Waldauf, A. J. Heeger and C. J. Brabec, Adv. Mater., 2006, 18, 789-794.

64 J.-L. Brédas, D. Beljonne, V. Coropceanu and J. Cornil, Chem. Rev., 2004, 104, 4971-5004.

65 M. E. Köse, J. Phys. Chem. A, 2012, 116, 12503-12509.

66 A. Dkhissi, Synth. Met., 2011, 161, 1441-1443.

67 B. G. Kim, C. G. Zhen, E. J. Jeong, J. Kieffer and J. Kim, Adv. Funct. Mater., 2012, 22, 1606-1612.

68 M. Ans, K. Ayub, S. Muhammad and J. Iqbal, Comput. Theor. Chem., 2019, 1161, 26-38.
69 S. K. Gangala, M. Paramasivam, B. Dyaga and V. J. Rao, New J. Chem., 2019, 43(13), 5173-5186.

70 V. Arkhipov, P. Heremans and H. Bässler, Appl. Phys. Lett., 2003, 82, 4605-4607.

71 C. Marchiori and M. Koehler, Synth. Met., 2010, 160, 643650.

72 M. Koehler, M. Santos and M. Da Luz, J. Appl. Phys., 2006, 99, 053702 .

73 S. Baranovskii, M. Wiemer, A. Nenashev, F. Jansson and F. Gebhard, J. Phys. Chem. Lett., 2012, 3, 1214-1221. 